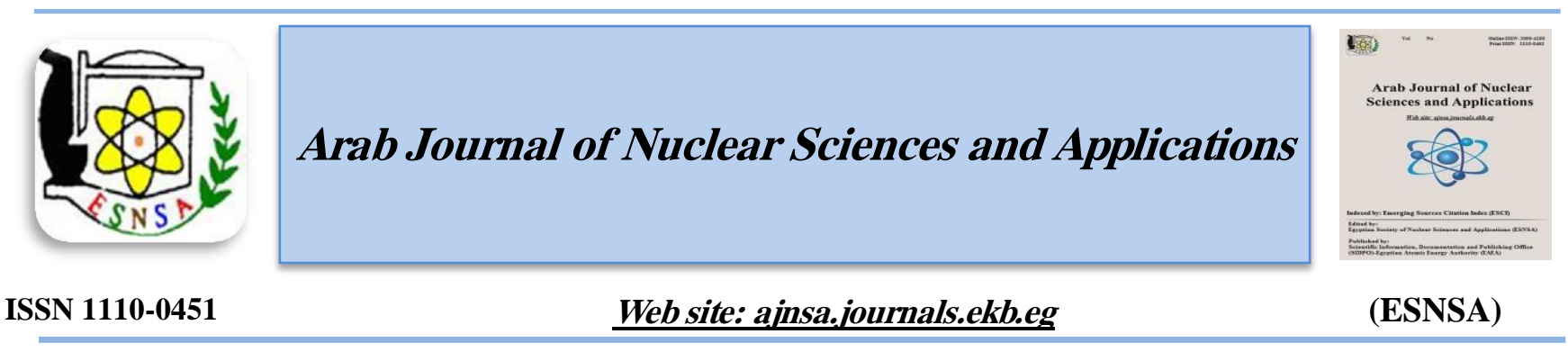

\title{
Peripheral Photon Doses from Different Techniques Delivered in Prostate Radiotherapy: Experimental Measurements and TPS Calculations
}

\author{
Hala A. Soliman ${ }^{a^{*},}$ Ranya M. Moussa ${ }^{\mathrm{b}}$, and Maha Kamaleldin ${ }^{\mathrm{b}}$ \\ ${ }^{a}$ Ionizing Radiation Metrology Lab., National Institute of Standards, Giza, Egypt \\ ${ }^{b}$ Radiation Therapy Department, Faculty of Medicine, Cairo University, Cairo, Egypt
}

Received $19^{\text {th }}$ Jun. 2019 Advanced techniques and modalities in delivering radiotherapy improved the ability of delivering Accepted $2^{\text {nd }}$ Dec. 2019 higher tumor doses while minimizing dose to the critical organs. The aim of this work is to determine experimentally the out-of-field doses associated with these modern radiotherapy techniques for the actual clinical case of prostate carcinoma and intercompare it with the treatment planning system (TPS) calculations. Three prostate treatment plans of the intensity-modulated radiation therapy (IMRT), Rapid Arc, and the Three-Dimensional Conformal Radiotherapy (3D-CRT) techniques were delivered to an anthropomorphic phantom using $6 \mathrm{MV}$ photons beams produced from a UNIQUE linear accelerator. The out-of-field surface doses at various distances from the isocenter and peripheral doses to organs at risk (OARs) were measured using the thermoluminescent detectors (TLD-700) then compared with the calculated doses from the used commercial Eclipse TPS version 11. The two implemented algorithms in the Eclipse TPS (analytical anisotropic algorithm, AAA and the Acuros XB algorithm, AXB) underestimated the surface out-of-field doses by a maximum average deviation of $49 \%$ till the distance of $15 \mathrm{~cm}$ from the isocenter reported to the 3D-CRT technique. IMRT and RA techniques were more precise than 3D-CRT in tumor coverage and sparing of OARs but resulted in larger peripheral photon doses. RA technique has the advantage of less treatment time than IMRT technique and also produces less peripheral photon doses because it uses fewer monitor units. Our results show that although AXB algorithm is significantly faster in dose calculations than $\mathbf{A A}$ algorithm especially in modern techniques, but it is less accurate in estimating the out-of-field doses.

Key words: Out-of-field doses; TLD-700; AAA algorithm; AXB algorithm; Prostate cancer

\section{Introduction}

Prostate cancer is the second most common cancer [1] and lethal tumor in men [2], however, it is a curable disease when it is diagnosed and treated in early stages. External beam radiotherapy (EBRT), is one option from the most common treatment options. EBRT includes the Three-Dimensional Conformal Radiotherapy (3D-CRT), the advanced techniques of the Intensity modulated Radiotherapy (IMRT) and Rapid Arc (RA) are used. These modern techniques increased the ability of delivering higher doses to the tumor while minimizing the dose to the adjacent organs at risk (OARs). Anna Kowalik et al [3] compared
3D-CRT, IMRT, and tomotherapy in assessing doses to OARs resulting from photon beam irradiation and scattered neutrons. They found that all OARs outside the therapeutic field are wellspread for tomotherapy while IMRT accomplished better sparing in only the bladder than 3D-CRT. The high doses of radiation delivered by these techniques may lead to long-term adverse effects and primarily second cancers as a consequence of the significantly larger out-of-field doses [4-8]. The patient receives the dose outside of the primary radiation field (out-of-field doses) due to secondary scattered radiation from the accelerator head, treatment room (the floor, walls, ceiling), the

Corresponding author: halasoliman4@yahoo.com

DOI: 10.21608/ajnsa.2019.13839.1222

(C) Scientific Information, Documentation and Publishing Office (SIDPO)-EAEA 
patient's own body, and/or leakage through the collimator $[9,10]$. Radiation leakage around the gantry comes from the electron beam guide and the head of the linear accelerator, so it could vary depending on the used type of linear accelerator (linac) even for the same manufacturer [11,12]. Treatment Planning Systems (TPSs) could not model these out-of- field doses at large distances from the field edge till now and its accuracy of dose estimation beyond a few centimeters outside the treatment field edge is usually poor $[10,13]$. Howell et al [13] found that the used Eclipse TPS underestimated out-of-field doses by an average of $40 \%$ for a treatment plan of historic mantle field.

The aim of the present work is to determine the peripheral photon doses resulting from prostate radiotherapy employing the 3D-CRT technique and to compare the results with the other two modern techniques of IMRT and RA, experimentally and theoretically. A realistic anthropomorphic RANDO phantom was used for the clinical treatments which is delivered on a UNIQUE linac (Varian Medical Systems Inc., UK) with $6 \mathrm{MV}$ photon beams. The treatment plans of the three different techniques were created in a commercially TPS, Eclipse version 11.0 (Varian Medical Systems, Inc., UK), with a prescription dose of one-shot [14] of 17.8 Gy to the isocenter of the field which is biologically equivalent dose of 76 Gy/38 fractions calculated with the linear quadratic model $[15,16]$.

Two algorithms were implemented in the Eclipse TPS, the Anisotropic Analytical Algorithm (AAA) and a new commercially available Acuros XB advanced dose calculation algorithm (AXB). The accuracy of the two used algorithms were evaluated and compared in estimating these peripheral photon doses.

\section{Material and Methods}

Dose calculation algorithms

The Eclipse TPS was supporting the AAA and AXB algorithms. AAA algorithm is a 3D-pencil beam convolution-superposition with two components, the configuration algorithm and the dose calculation algorithm. It uses pre-computed Monte Carlo simulated beam data and scatter kernels $\mathrm{K}$ to calculate the absorbed dose in a medium. The configuration algorithm assesses the physical parameters of the beam (fluence and energy spectra), and their scattering properties in water-equivalent medium. The primary photons, scattered photons and electrons from the beam limiting devices are modeled separately [17]. The dose calculation algorithm determines the heterogeneity of the medium to provide a consistent estimation of dose distribution in the plans [18] This heterogeneity is managed by scaling of primary photons and photon scatter kernel scaling in lateral directions according to local electron density $[17,19,20]$.

The AXB algorithm was recently implemented in the Eclipse TPS and was developed to produce faster and more accurate dose distribution in external photon beam treatment planning. The AXB solves numerically the Linear Boltzmann Transport Equation which describes the macroscopic behavior of radiation particles as they travel through and interact with matter [21]. It calculates the dose in the medium based on energy deposition in a way similar to Monte Carlo especially in modeling of dose deposition in heterogeneous media. It is also insensitive to the number of calculated fields and consequently suitable for treatment planning of IMRT and RA techniques [22,23]. Aydin Cakir [24] investigated the dose-related effect of dose calculation grid size (CGS) for both AAA and AXB algorithms and they found afterward that AXB algorithm provided more stable results than the AAA algorithm in different intensity body regions.

\section{Treatment planning}

Anthropomorphic Alderson Rando phantom (Radiology Support Devices Inc., CA, USA) of $175 \mathrm{~cm}$ tall and $73.5 \mathrm{Kg}$ weight was used. The whole Rando phantom Fig. (1) was Computer Tomography (CT)-scanned, and 3 treatment plans simulating the prostate cancer using the 3 different techniques of IMRT, Rapid Arc, and 3-D CRT were generated from the Eclipse TPS (from Varian Medical Systems Inc., UK) version 11.0. The treatment plans were calculated by the two implemented commercially AAA [18] and the AXB [23] algorithms with a grid size of $2.5 \mathrm{~mm}$. The IMRT plan used 9 equi-spaced co-planar fields with angles starting from $180^{\circ}$ around the patient with machine dose rate of $300 \mathrm{cGy} / \mathrm{min}$. The RA plan used 2 full arcs with posterior avoidance sector of $30^{\circ}$ to $40^{\circ}$ in the rectal region, with a collimator angle $30^{\circ}$ and dose rate of 600 cGy/min. The constraints used in optimization are fixed for both IMRT and RA. For conformal planning, 5 co-planner fields are used and 
positioned in fan shape (anterior, 2 lateral wedged fields, and 2 anterior oblique fields with weight of $0.6,1$, and 0.7 respectively) Fig. (2).

\section{Phantom irradiation}

The RANDO phantom consists of 34 slices each of $2.5 \mathrm{~cm}$ thickness. Each slice has holes for accommodating the Thermoluminescent Detectors (TLDs); the TLD detectors are $4.5 \mathrm{~mm} \times 0.9 \mathrm{~mm}$ disks of TLD-700 (LiF; Mg, Ti) crystals (Harshaw Chemical Company, Solon, OH). For each measurement point, three TLD chips were implanted into the predrilled holes in the phantom slabs and on the surface of the phantom (along its main axis) every $5 \mathrm{~cm}$ increment till $60 \mathrm{~cm}$ away from the isocenter, yielding three readings which were averaged to obtain the TLD-measured dose for every measurement point. The phantom was delineated as a prostate case. PTV (planning target volume) is defined as the prostate and seminal vesicles with a $0.5 \mathrm{~cm}$ posterior margin and $1 \mathrm{~cm}$ margin in the other directions. Risk structures (OARs organs) such as bladder wall, rectal wall was also delineated.

The phantom was irradiated on the linear accelerator Unique (Varian Medical Systems Inc., UK) at the Radiation Therapy Department-Faculty of Medicine, Cairo University, Egypt. The linac has a millennium MLC-120 offering $0.5 \mathrm{~cm}$ leaf resolution at isocenter for the central $20 \mathrm{~cm}$ of the $40 \mathrm{~cm} \mathrm{x} 40 \mathrm{~cm}$ field. It produces photon energy of $6 \mathrm{MV}$ and the dose rate was up to $600 \mathrm{MU} /$ minute. The prescribed photon dose was the same for the three types of therapy, it was a one-shot dose of 17.8 Gy which is biologically equivalent to 76 Gy/38 fractions, calculated with the linear quadratic model. This dose was used to reduce significantly the beam-on time and to avoid linac overheating. The total number of monitor units (Mus) required to deliver this photon dose for IMRT, RA, and 3D-CRT were 6104, 6095, and 2976 respectively.

\section{TLD analysis}

TLD dosimeters underwent calibration, read-out and analyzed at the Radiation Therapy Department-Faculty of Medicine, Cairo University, as part of their quality assurance protocol. The dosimeters were calibrated in order to obtain their calibration factors from the same used linac (the UNIQUE) for Rando irradiation. After the irradiation had been delivered, the TLD dosimeters were read after 24 hours using the automatic RADOS RE-2000 TLD reader. Then, the background and individual correction factors were applied for each detector. The TLD system uncertainty of dose calculation was $3.25 \%$.

\section{Results}

Table (1) shows the measured (by TL-700) out-offield scattered doses as a relative percentage for the prescribed dose $(17.8 \mathrm{~Gy})$ at the isocenter of the field for the three delivered planning techniques (IMRT, RA, and 3D-CRT) in treating prostate carcinoma by $6 \mathrm{MV}$ photon beams.

Figure (3) shows the measured out-of-field surface doses for the three techniques in $5 \mathrm{~cm}$ increments away from the central axis of the field (field size of approximately $10 \times 10 \mathrm{~cm}^{2}$ ) till $60 \mathrm{~cm}$. The measured out-of- field dose distribution for the three techniques show the same exponential behavior.

Differences between the three measured techniques were similarly small especially at different distances except at the field edge (distance of 5 $\mathrm{cm}$ ), where the 3D-CRT technique shows a higher value than both of IMRT and RA techniques. The IMRT out-of- field dose profile shows higher scattered peripheral doses for almost all our measurement points.

Figures (4, 5 and 6) show plots of the measured and calculated (by AAA and AXB algorithms implemented in the Eclipse TPS) out-of-field doses for the delivered treatment techniques of IMRT, RA and 3D-CRT respectively. The Two algorithms underestimated the peripheral scattered doses till a distance of $19 \mathrm{~cm}$. But they did not follow the exponential falloff behavior of the measured out-of-field doses, and become more erratic especially after a distance of $15 \mathrm{~cm}$. At 15 $\mathrm{cm}$ distance the AAA algorithm was more accurate than AXB algorithm and estimated the out-of-field doses by an average of $10 \%$ in comparison with the AXB (33\%) using the IMRT Technique. The largest average deviation was $49 \%$ for the AXB algorithm in comparison with the AAA algorithm (24\%) when applying the 3D-CRT technique. At a distance of $19 \mathrm{~cm}$ from the isocenter there was a maximum deviation of $99 \%$ and $69 \%$ for the AAA and $\mathrm{AXB}$ algorithms respectively reported for the 3D-CRT technique. For further distances beyond the $19 \mathrm{~cm}$, the TPS results were not possible. The priority of the treatment plan is to apply the maximum dose to the tumor based on constraints 
of surrounding organs at risk [25]. Table (2) shows the measured sparing of adjacent sensitive organs (Bladder and Rectum), and their estimation by the used algorithms for the different techniques as an equivalent dose of $\mathrm{mSV}$ per unit photon Gy delivered to the isocenter. Our results of TLD measurements show an advantage of RA technique over IMRT and 3D-CRT techniques for localized prostate cancer in regard to dose sparing of OARs and the least required monitor units (MUs). AAA algorithm estimated doses were in good agreement with the measurements in all of the used modalities by an average of $2.62 \%$, while the AXB estimation results show underestimation by an average of $4.43 \%$. It is noticed that the AAA and AXB algorithms estimations show better results with the 3D-CRT technique than the other two techniques.

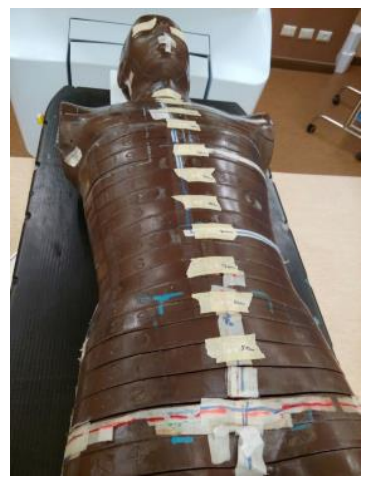

Fig. (1): The whole RANDO phantom used for TLD measurements

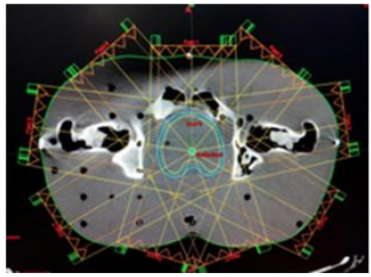

(a)

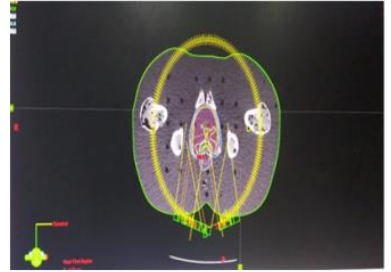

(b)

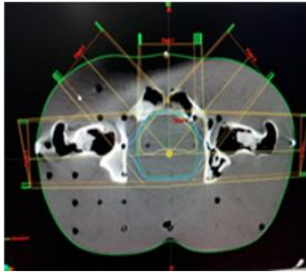

(c)

Fig. (2): Representative axial CT slices showing the arrangement of fields for (a) IMRT, (b) RA, and (c) 3D-CRT techniques

Table (1): Measurements of low scattered out-of-field doses by TLD-700 dosimeters (as a relative percentage for the 17.8 Gy prescribed dose at the isocenter of the field) by 3 different techniques (IMRT, Rapid Arc and 3D-CRT)

\begin{tabular}{c|c|c|c}
\hline $\begin{array}{c}\text { Distance } \\
\text { from } \\
\text { isocenter } \\
(\mathrm{cm})\end{array}$ & IMRT & Rapid Arc & 3D-CRT \\
\cline { 2 - 4 } & \multicolumn{3}{|c}{ Values in \% } \\
\hline 5 & 2.955 & 2.961 & 3.045 \\
10 & 1.348 & 1.292 & 1.281 \\
15 & 0.826 & 0.792 & 0.775 \\
20 & 0.410 & 0.393 & 0.376 \\
25 & 0.320 & 0.298 & 0.265 \\
30 & 0.180 & 0.185 & 0.152 \\
35 & 0.169 & 0.174 & 0.146 \\
40 & 0.104 & 0.107 & 0.101 \\
45 & 0.064 & 0.064 & 0.053 \\
50 & 0.040 & 0.039 & 0.029 \\
55 & 0.038 & 0.037 & 0.024 \\
60 & 0.033 & 0.030 & 0.023 \\
\hline
\end{tabular}




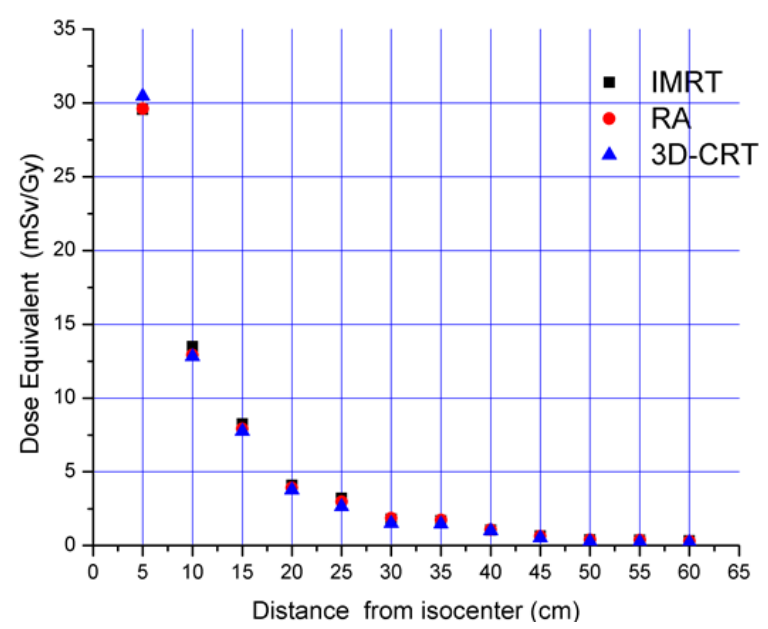

Fig. (3): Measured out-of-field surface dose profiles by TLD-700 along the main axis of the RANDO phantom for the three treatment techniques

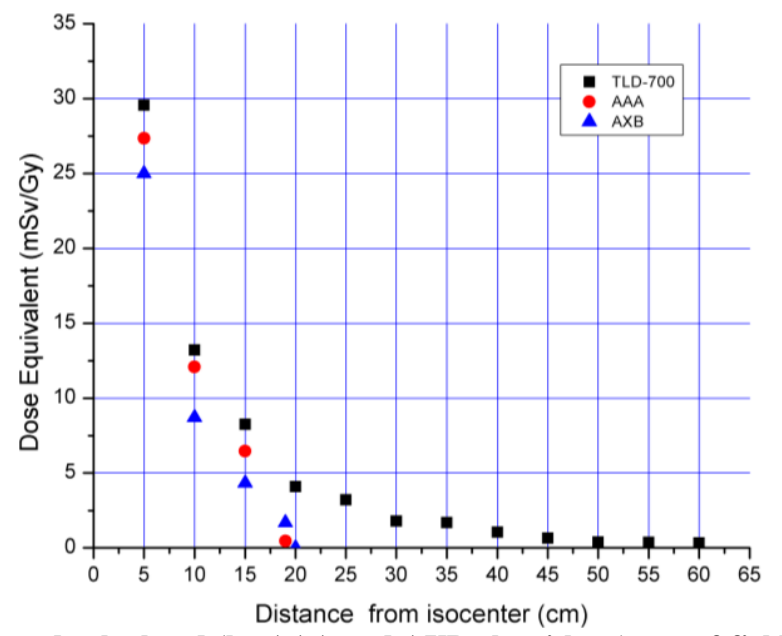

Fig. (4): Measured (by TLD-700) and calculated (by AAA and AXB algorithms) out-of-field surface dose profiles for IMRT technique

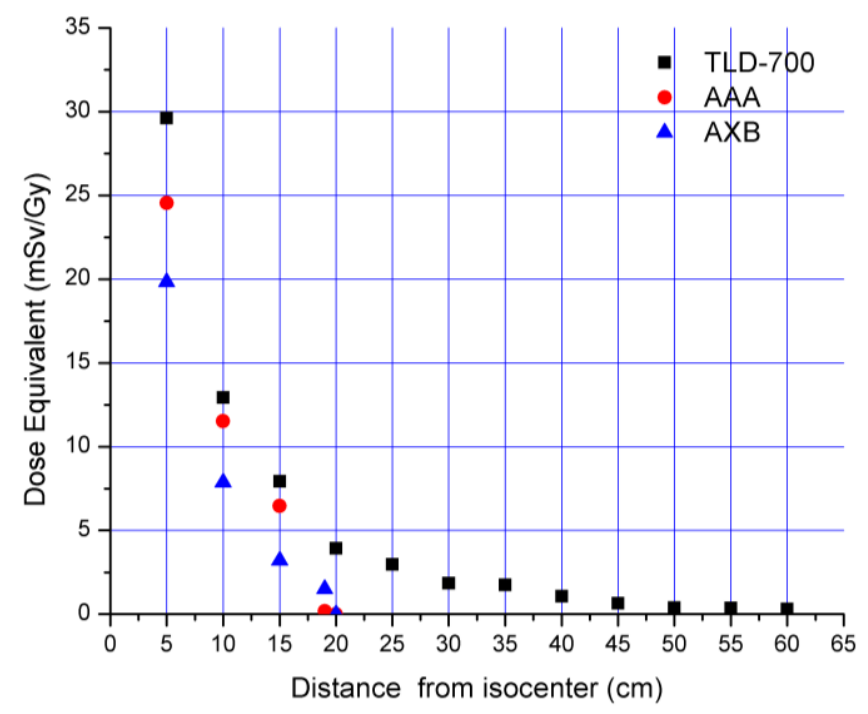

Fig. (5): Measured (by TLD-700) and calculated (by AAA and AXB algorithms) out-of-field surface dose profiles for RA technique 


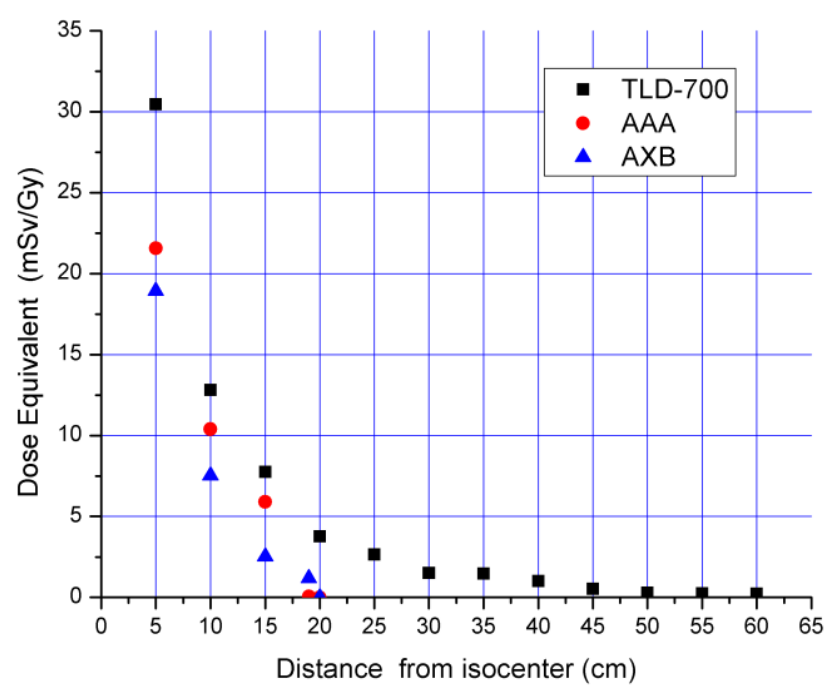

Fig. (6): Measured (by TLD-700) and calculated (by AAA and AXB algorithms) out-of-field surface dose profiles for 3DCRT technique

Table (2): Measured and calculated equivalent doses (in mSv per unit photon Gy at the isocenter) to OARs delivered from the used three different techniques

\begin{tabular}{|c|c|c|c|c|c|c|c|c|c|}
\hline \multirow{3}{*}{$\begin{array}{l}\text { Organs } \\
\text { at Risk }\end{array}$} & \multicolumn{7}{|c|}{ Delivered Technique } & & \\
\hline & \multicolumn{3}{|c|}{ IMRT } & \multicolumn{3}{|c|}{ RapidArc } & \multicolumn{3}{|c|}{ 3D-CRT } \\
\hline & TLD-700 & AAA & $\mathrm{AXB}$ & TLD-700 & AAA & $\mathrm{AXB}$ & TLD-700 & AAA & $\mathrm{AXB}$ \\
\hline Bladder & 7.98E-01 & $7.80 \mathrm{E}-01$ & $7.56 \mathrm{E}-01$ & 7.84E-01 & 7.61E-01 & 7.44E-01 & $8.71 \mathrm{E}-01$ & $8.45 \mathrm{E}-01$ & 8.33E-01 \\
\hline Rectum & 8.99E-01 & $8.75 \mathrm{E}-01$ & $8.59 \mathrm{E}-01$ & 8.34E-01 & 8.12E-01 & 8.03E-01 & $9.5 \mathrm{E}-01$ & $9.32 \mathrm{E}-01$ & $9.20 \mathrm{E}-01$ \\
\hline
\end{tabular}

\section{Discussion}

Comparisons of out-of-field doses for different treatment planning techniques to prostate carcinoma using the RANDO phantom and TLD700 (with 6 MV photons) show differences between them in producing these low scattered doses. The results are similar to those of conventional field data from the report of AAPM Task Group 36 [25], as reported by many authors, confirmed the decrease in dose as distance from the edge of the therapeutic field increases $[10,26,27,12]$. The 3D-CRT technique shows higher scattered dose compared to the other two techniques at the field edge $(5 \mathrm{~cm}$ distance), because of the patient scatter which is the main component near the field.

The RA and IMRT techniques show higher low peripheral doses than the 3D-CRT technique because of the collimator scatter and head leakage which dominate the picture for $15-30 \mathrm{~cm}$ distance from the field edge [28, 29, 30]. The IMRT technique shows the higher scattered dose Arab J. Nucl. Sci. \& Applic. Vol. 53, No. 1 (2020) especially at distances far away from the field edge because of the head leakage scatter component result from the field modulation and increasing more monitor units compared with delivering the same dose from an unmodulated field [31,32].

In the present study, the measured out-of- field surface doses were calculated by the Eclipse TPS for the different delivered techniques for the clinical treatment of prostate with the Unique linac.

The results Figs. (4, 5, and 6) show underestimation to the actual dose by an average of $10 \%, 15 \%$, and $24 \%$ for the IMRT, RA, and 3DCRT techniques; respectively; using the AAA algorithm. On the other hand, the AXB algorithm shows more underestimation of $33 \%, 44 \%$, and $49 \%$ for IMRT, RA, and 3D-CRT respectively. The results also show that this underestimation get worse for distances far away from the isocenter of the field. This decreased accuracy, noted by the three techniques for the two used algorithms are attributed to the underestimation of the collimator 
scatter and patient scatter near the field edge and the underestimation of the head leakage at large distances from the treatment field.

AAA algorithm was more accurate than the AXB algorithm in estimating the out-of-field doses for all of the used planning techniques because of the different method in dose calculation. The AAA algorithm models extra-focal photon radiation using a finite-size virtual source (the second source) which has a Gaussian intensity distribution. The second source energy fluence is defined at an arbitrary plane and is computed by summing the contributions from each element of the source for each pixel in the destination fluence array (Eclipse AAA manual). Plan dose calculation in Acuros XB calculate the scattered photons and electron fluence once for all fields in the plan. As a result, the relative calculation speed of AXB increases with increasing numbers of fields in the plan. So AXB algorithm is significantly faster in dose calculations than AAA algorithm especially in advanced treatment planning techniques of multiple fields like RA and IMRT, but less accurate.

For prostate cancer, the organs at risk are the bladder and the rectum. Minimizing the dose to these organs is a mandatory part of the treatment planning process [24]. Comparing the three planning techniques with respect to their plan quality and treatment efficiency (dose homogeneity within the PTV, and minimized dose to the surrounding normal tissues and critical organs) result in advantage of RA and IMRT techniques over the 3D-CRT technique in regard to dose sparing of OARs. Also, the RA technique has the advantage of less treatment time and the use of fewer MUs than the IMRT technique.

\section{Conclusion}

From the present study, some general conclusions can be drawn in the light of the above discussion. First, measuring the out-of-field doses (by TLD700) produced from different modern modalities in prostate radiotherapy by $6 \mathrm{MV}$ photons of Unique linac, result in an exponential dose distribution curves that decrease with greater distances from the isocenter. IMRT technique shows higher scattered low doses for almost all our measurement points because of the increase number of monitor units and consequently the increase in collimator scatter and head leakage of the machine. Second, using the Eclipse TPS and its implemented AAA and $\mathrm{AXB}$ algorithms in estimating these out-offield doses, show an underestimation of different values for the three techniques with a maximum average estimation of $49 \%$ for the 3D-CRT technique calculated by the AXB algorithm. Far from the isocenter, the TPS errors of calculation approaches 100\%. Third, although IMRT technique produces the higher out-of- field doses but it has an efficient plan quality and the ability to minimize dose to critical normal structures. The RA technique has also an efficient plan quality and dose sparing to OARs with the advantage of using fewer Mus than IMRT technique and less treatment time.

3D-CRT technique shows good target coverage and the lowest out of-field scattered doses, but resulted in higher doses to OARs. Eclipse TPS generated treatment plans for the three techniques were in good agreement with the measurements especially the 3D-CRT technique. The AXB algorithm is significantly faster in dose calculation than the AAA algorithm especially in advanced treatment planning techniques of multiple fields like RA and IMRT but less accurate especially in estimating out-of-field doses.

\section{References}

1. Jemal A, Bray F, Center MM, Ferlay J, Ward E, Forman D (2011) Global cancer statistics. CA Cancer J Clin; 61:69-90.

2. Cooperberg MR, Moul JW, Carroll P (2005) The changing face of prostate cancer. Journal of Clinical Oncology; 23: 8146 - 8156.

3. Anna Kowalik, Jackowiak W., Malicki J., Skórska M., Adamczyk M., Konstanty E., Piotrowski T., Polaczek-Grelik K. (2017) Measurements of doses from photon beam irradiation and scattered neutrons in an anthropomorphic phantom model of prostate cancer: a comparison between 3DCRT, IMRT and tomotherapy. NUKLEONIKA 2017;62(1):29-35.

4. Cho S, Kim SH, Kim CH, Park JG, Park JH, Jeong JH. (2011) Secondary cancer risks in out-of-field organs for 3-D conformal radiation therapy. Prog Nucl Sci Technol; 1:521-4.

5. Howell RM, Hertel NE, Wang Z, Hutchinson J, Fullerton GD (2006) Calculation of effective dose from measurements of secondary neutron spectra and scattered photon dose from dynamic MLC IMRT for $6 \mathrm{MV}, 15 \mathrm{MV}$ and 18 MV beam energies. Med Phys; 33: 360-8.

6. Kry SF, Price M, Followill D, Mourtada F, Salehpour M (2007). The use of LiF (TLD- 
100) as an out-of-field dosimeter. Med Phys; 8:2679.

7. Bednarz B, Xu XG. (2009) Monte Carlo modelling of a 6 and 18 MV Varian Clinac medical accelerator for in-field and out-of-field dose calculations: development and validation. Phys Med Biol;54: N43-57.

8. Zemanova M, Machalekova K, Sandorova M, Boljesikova E, Skultetyova M, Skultetyova, Sevec J, and emane A (2014) Clinical management of secondary angiosarcoma after breast conservation therapy. Rep Pract Oncol Radiother; 19:37-46.

9. Benadjaoud M, Bezin J, Veres A, Lefkopoulos D, Chavaudra J, Bridier A, Vathaire F, Diallo I (2012) A multi-plane source model for out-offield head scatter dose calculations in external beam photon therapy. Phys Med Biol; 57: 7725-39.

10. Huang JY, Followill DS, Wang XA, Kry SF (2013). Accuracy and sources of error of outof-field dose calculations by a commercial treatment planning system for intensitymodulated radiation therapy treatments. J Appl Clin Med Phys.; 14:186-197.

11. Lonski P, Taylor ML, Franich RD, Harty P, Kron T (2012). Assessment of leakage doses around the treatment heads of different linear accelerators. Radiat Prot Dosimetry; 152:30412.

12. M. Kruszyna, S. Adamczyk, A. Skorska, W. Suchorska, K. Zaleska, A. Kowalik, W. Jackowiak, J. Malicki (2017) Low dose out-offield radiotherapy, part 1: Measurement of scattered doses. Cancer Radiother 21 (5): 345 351.

13. Howell RM, Scarboro SB, Kry SF, Yaldo DZ (2010) Accuracy of out-of-field dose calculations by a commercial treatment planning system. Phys Med Biol.; 55:6999_ 7008 .

14. Thomas Zilli1, Marta Scorsetti, Daniel Zwahlen, Ciro Franzese, Robert Förster, Niccolò Giaj-Levra, Nikolaos Koustouvelis, Aurelie Bertaut, Michel Zimmermann, Giuseppe Roberto D’Agostino, Filippo Alongi, Matthias Guckenberger and Raymond Miralbell (2018) ONE SHOT -single shot radiotherapy for localized prostate cancer: study protocol of a single arm, multicenter phase I/II trial. Radiation Oncology (2018) 13:166

15. J. F. Fowler (1989) The linear-quadratic formula and progress in fractionated radiotherapy. Br. J. Radiol. 62,679-694.

16. Brenner, D. J., Hlatky, L. R., Hahnfeldt, P. J., Huang, Y. and Sachs, R. K (1998) The LinearQuadratic Model and Most Other Common
Radiobiological Models Result in Similar Predictions of Time-Dose Relationships. Radiat. Res. 150,83-91.

17. Tillikainen L, Helminen H, Torsti T, Siljamäki S, Alakuijala J, Pyyry J, Ulmer W (2008) A 3D pencil-beam-based superposition algorithm for photon dose calculation in heterogeneous media. Phys Med Biol.; 53: 3821-39.

18. Sievinen, J.; Ulmer,W.; Kaissl,W. AAA photon dose calculation model in Eclipse ${ }^{\mathrm{TM}}$. Varian Medical Systems: 18.

19. Ulmer W, Harder D. (1996) Applications of a triple gaussian pencil beam model for photon beam treatment planning. Med Phy. 6:68-74.

20. Van Esch A, Tillikainen L, Pyykkonen J, Tenhunen $M$, Helminen $H$, Siljamäki S, Alakuijala J. (2006) Testing of the analytical anisotropic algorithm for photon dose calculation. Med Phys.; 33: 4130-48.

21. Vassiliev ON, Wareing TA, McGhee J, Failla G, Salehpour MR, Mourtada F (2010) Validation of a new grid based Blotzmann equation solver for dose calculation in radiotherapy with photon beams. Phys Med Biol.; 55: 581-98.

22. Suresh Rana, Kevin Rogers, Shyam Pokharel, Terry Lee, Daniel Reed and Christophr Big (2013) Acuros XB algorithm vs. anisotropic analytical algorithm: A dosimetric study using heterogeneous phantom and computed tomography (CT) data sets of esophageal cancer patients. J. Cancer Ther. 4:138-

23. Failla, G.A., Wareing T., Archambault Y., Thompson S. Acuros XB advanced dose calculation for the Eclipse treatment planning system. Acuros XP clinical prospective. Varian Medical Systems: 32.

24. ÇAKIR A. (2017) Dosimetric Comparison of Anisotropic Analytical Algorithm and Acuros $\mathrm{XB}$ in Stereotactic Body Radiotherapy and Effect of Calculation Grid Size. Turk J Oncol 2017;32(3):100-105.

25. Stovall M, Blackwell CR, Cundiff J, Novack DH, Palta JR, Wagner LK, Webster EW, Shalek RJ. (1995) Fetal dose from radiotherapy with photon beams: report of AAPM Radiation Therapy Committee Task Group No. 36. Med Phys. 22(1):63-82.

26. Miljanic, S., Bessieres, I., Bordy, J.M., d'Errico, F., Di Fulvio, A., Kabat, D., Knezevi_c, ZZ., Olko, P., Stolarczyk, L., Tana, L., Harrison, R. (2013). Clinical simulations of prostate radiotherapy using BOMAB-like phantoms: results for photons. Radiat. Meas. 57, 35-47.

27. Ruben JD, Lancaster CM, Jones P, Smith RL (2011) A comparison of out-of-field dose and its constituent components for intensity- 
modulated radiation therapy versus conformal radiation therapy: implications for carcinogenesis. Int J Radiat Oncol Biol Phys. 81:1458-1464.

28. Ruben JD, Davis S, Evans C, Jones P, Gagliardi F, Haynes M, Hunter A (2008). The effect of intensity-modulated radiotherapy on radiation-induced second malignancies. Int $\mathbf{J}$ Radiat Oncol Biol Phys. 70:1530-1536.

29. Kase KR, Svensson GK, Wolbarst AB, Marks MA (1983) Measurements of dose from secondary radiation outside a treatment field. Int J Radiat Oncol Biol Phys; 9: 1177-1183.

30. Lillicrap SC, Morgan HM, Shakeshaft JT (2000) X-ray leakage during radiotherapy. Br J Radiol 73: 793-794.

31. Hall EJ, Wuu CS. (2003) Radiation-induced second cancers: the impact of 3D-CRTand IMRT. Int J Radiat Oncol Biol Phys;/56 (1): /83-/88.

32. Williams PC, Hounsell AR (2001) X-ray leakage considerations for IMRT. Br J Radiol; 74:98-102. 